\title{
Ariadne 1.0: A Novel Method and Prototype for Artificially Spinning Spider Silk
}

\author{
Avery Williamson ${ }^{1}$, Haley Bergman ${ }^{1}$, Preston Vanderpan ${ }^{1}$ and Marc Facciotti ${ }^{\#}$ \\ ${ }^{1}$ University of California, Davis, CA, USA \\ "Advisor
}

$\underline{\text { ABSTRACT }}$

Musculoskeletal conditions affect more than half of the U.S. population over 18 years old, accounting for more than $50 \%$ of all disabling health conditions in adults [1]. The push for innovative surgical technologies has sparked a search for new biomaterials analogous to, or better than, native human musculoskeletal tissue. Spider silk is five times the tensile strength of steel by weight, as elastic as rubber on a weight-to-weight basis, antibacterial, conductive, and hypoallergenic [2]. Applications of this unique biomaterial include artificial tissues such as tendons, ligaments, skin grafts, muscles, and neurons. The 3D Organic Polymer Silk team (3D-OPS) has designed a cost-effective, novel system, Ariadne 1.0, to manufacture 3D printed spider silk as an alternative to biomaterials currently used in medical applications. The pre-printing process of Ariadne 1.0 utilizes native spider silk or purified spidroin proteins in the production of a stable solution for use in 3D printing of biological structures. Modeled after the chemico-physical environment of the Nephila clavipes' major ampullate gland, Ariadne 1.0's printing system can easily modify the biophysical properties of its silk product by altering parameters such as the printing solution's protein-type, ion concentration, or weight to volume ratio and the printing system's needle size and print speed. This versatility supports a broad spectrum of surgical procedures, and enables potential for improvements in the medical field, particularly surrounding musculoskeletal injuries. The 3D-OPS team designed Ariadne 1.0 to investigate the viability of printing artificial tendons, ligaments, and muscle fibers with spider silk.

\section{Description of the Problem}

More than half of the U.S. population over 18 years old are affected by musculoskeletal conditions, amounting to more than $50 \%$ of all disabling health conditions in adults [1]. Existing materials used in tissue construction and repair as an option in musculoskeletal treatments fail to restore damaged tissue's functional, structural, and biochemical properties [3]. Biomedical engineers require scaffolds capable of supporting vital cell activities such as adhesion, migration, proliferation, and differentiation [3], yet synthetic and natural scaffolding technologies do not meet these requirements. Natural scaffolds, such as allografts, autografts, and collagen generally require two surgical procedures, incorporate slowly into native structures, and experience deterioration of the original mechanical properties over time [6]. 2D and 3D scaffolds made from hard plastics or glass surfaces, while non-toxic and non-immunogenic, are incapable of supporting desired cell activity and pose a severe risk when integrated with native tissue [4]. Moreover, use of synthetic material and grafts can cause biophysical complications during the post-surgical integration period, leading to unexpected out-of-network fees between 10 to $40 \%$ of the original cost [5] and additional expenses for both patient and provider.

In the hunt to find a human tissue analog, scientific interest in spider silk and its potential applications has markedly intensified - specifically, native (unaltered by denaturing) spider silk's applicability to musculoskeletal injuries. The cannibalistic nature of spiders nullifies the possibility of collecting silk through traditional farming techniques, prompting the construction of artificial spinning systems. However, currently manufactured spider silk exhib- 
its poor mechanical performance. The process has low reproducibility and quality control, and manufacturing techniques require expensive lab equipment and caustic chemical reagents. Consequently, previous prototypes have been impractical for large scale implementation.

The lack of parametric control in the core processes of native silk formation restricts the production of affordable, high-quality artificial spider silk on an industrial scale. Revolutionizing musculoskeletal treatments hinges on discovering and implementing a novel biomaterial like spider silk to provide patients with full mechanical and biological capabilities, safer treatment options, and quicker recoveries.

\section{Project Objective Statement}

The 3D-OPS team has designed an affordable technique to 3D print spider silk. Ariadne 1.0 will allow for a less expensive and more efficient synthesis of biomaterials such as tendons, ligaments, and muscle fibers, as well as scaffolds and grafts. Spider silk is a superior material for synthetic tissue because it is nonirritating, has immense tensile strength, and is biocompatible - circumventing rejection reactions. Furthermore, the inherent differences between various silks introduce the possibility of designing composites with unique properties for advanced medical applications. Ariadne 1.0's pre-printing process will transform spidroin proteins with conserved C-terminal (CT) and Nterminal (NT) domains into a stable printing solution. The printing system of Ariadne 1.0 will mirror the chemicophysical environment of the Nephila clavipes' major ampullate gland transforming the solution into a 3D printed substance with similar properties to native spider silk. The design of Ariadne 1.0 allows users to control and modify the silk product's properties to physician- and patient- specific needs. The innovative design of Ariadne 1.0 will accomplish this by using low-cost, commercially available hardware and standard biological processes, making it easy to implement in any laboratory environment.

\section{Documentation of the Design}

A preliminary analysis of existing artificial silk-production techniques identified several possible shortcomings caused by the inadequate imitation of the natural spinning process [7]. Firstly, fiber formation by denaturation, aggregation, and coagulation relies on the repetitive domains of specific species and glands, requiring novel processes for each type of silk that amount to exorbitant costs. Ariadne 1.0 solves this problem by regulating conserved N-terminal and $\mathrm{C}$-terminal domains in spidroin proteins, eliminating the limitation of exclusively intra-species protein alignment. This allows us to mirror the enzymatic process of a spider gland and enable a single machine to produce a diversity of silks. Secondly, the most common of the traditional systems, the coagulation bath, involves injecting dope into a bath of costly, carcinogenic, extremely alkaline or acidic chemicals, and wet spinning [7]. The open structure of chemical baths prohibits the tapering or pressure manipulation required for silk uniformity; a single incorrect variable requires an entirely new system. In contrast, Ariadne 1.0 consists of uniform flexible tubing typically used for intravenous lines scaled to replicate the Nephila clavipes' major ampullate gland allowing the user to modify the preexisting system for specific alterations of the numerous chemico-physical variables; this maximizes efficiency and reduces costs. Lastly, current technology used in alternative silk-spinning systems with higher variable controls and tissue engineering remains hazardous, difficult to modify, and costly, ranging from approximately $\$ 10,000$ to $\$ 200,000$ per device, making it inaccessible for many institutions and research groups [8]. Ariadne 1.0 provides a more affordable choice by using common chemicals, readily available materials, and a modified filament printer to offer greater versatility and ease of modification.

\section{Pre-Printing Process and Dope Composition}


Ariadne 1.0's method for solubilizing spider silk is being designed for compatibility with orb-weaving spider spidroin proteins or those that conserve $\mathrm{C}$-terminal and $\mathrm{N}$-terminal domains, supporting modification of inputs to alter the characteristics of the end product [7]. The fabrication of the printing gel from the silk proteins has two possible inputs, native silk webs and purified spidroin proteins (transgenic or native). Preparation of the printing solution from native silk includes two additional steps, cleaning, and degumming, which precede the purified spidroin proteins process and begins with dissolution followed by adding a buffer, stabilizing proteins, and ending with the addition of a chemical chaperone.

Cleaning starts with washing the native silk and then soaking it overnight in distilled water to remove debris. The silk will dry in an oven at $60^{\circ} \mathrm{C}$ for one to two hours, then be degummed through immersion in a $0.02 \mathrm{M}$ sodium carbonate aqueous solution, stirring for $20-60$ minutes at $100^{\circ} \mathrm{C}$ to remove the tightly bound shell layer. After degumming, rinsing, and drying, the fiber will be ready for protein extraction. The purified protein process will begin with the dissolution of $30 \mathrm{mg}$ of degummed silk fiber in $60 \mathrm{~mL}$ of Ajisawa's reagent (calcium chloride: ethanol: water with a 1:2:8 molar ratio) with optimal conditions of $250 \mathrm{rpm}$, for 2.5 hours at $70^{\circ} \mathrm{C}$. Due to the NT and CT conservation of the spidroins, stir speed and temperature will be held constant [7, 9]. However, incubation time may deviate, falling between 2.5 to 5 hours. After dissolution, $\mathrm{NaHCO}_{3} / \mathrm{Na}_{2} \mathrm{CO}_{3}$ buffer with a concentration of $25 \mathrm{mM} \mathrm{HCO}_{3}{ }^{-}$will be added to the system, sustaining the $\mathrm{pH}$ gradient by mimicking the $\mathrm{pCO}_{2}$ and $\mathrm{HCO}_{3}{ }^{-}$concentrations in the native gland and facilitating the release of the CT domain (Figure 1) [10].

\section{Synthetic Ampullate Gland}

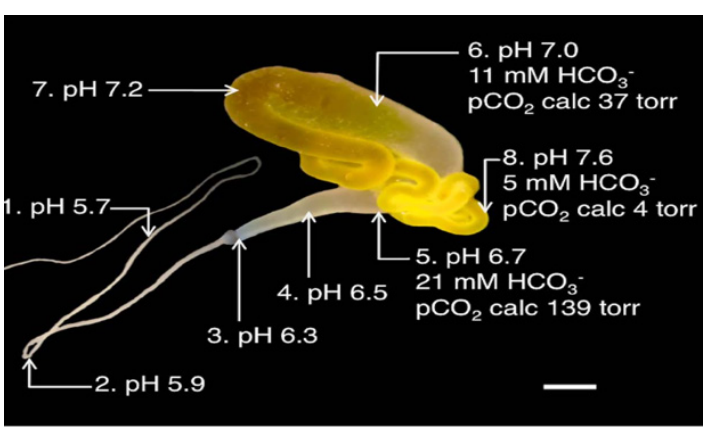

Figure 1. Map of Nephila clavipes' native gland and pH gradient [10].

Figure 2 shows the titration curve for the $\mathrm{NaHCO}_{3} / \mathrm{Na}_{2} \mathrm{CO}_{3}$ buffer and how it reacts between a $\mathrm{pH}$ range of A to $\mathrm{B}$ (7.2 to 5.7) [11]. Following the buffer solution, 0.1-0.2 $\mathrm{M}$ sodium chloride will be added to reach a sodium concentration of $199 \mathrm{mM}$ and a chloride concentration of $164 \mathrm{mM}$, mirroring Table 1's combination of the native duct's concentrations and preventing the early aggregation of proteins. Then $0.25-0.75 \mathrm{M}$ urea will be added as a chemical chaperone, ensuring no premature protein folding [17]. The use of urea also aids in establishing a stable aqueous solution of recombinant spider silk proteins that match native spider silk proteins' $30-50 \% \mathrm{w} / \mathrm{v}$ ratio. The resulting solution should have a $\mathrm{pH}$ of $\approx 7.3-7.0$, which matches the $\mathrm{pH}$ value of the native spinning sac. This reinforces the stability of the solution, and enables mass production and the ability to maintain a stock solution without risking degradation or aggregation of the proteins. 


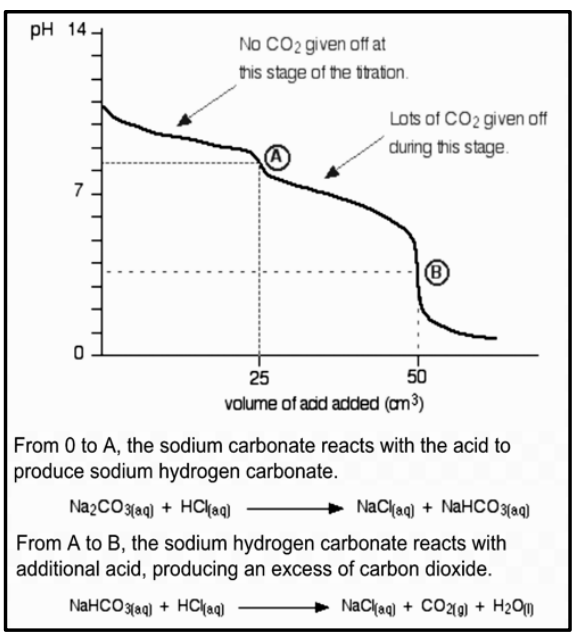

Figure 2. Titration curve for the $\mathrm{NaHCO}_{3} / \mathrm{Na}_{2} \mathrm{CO}_{3}$ buffer

Table 1. Native Duct Concentrations of Nephila clavipes [10, 12, 13, 14, 15, 16]

\begin{tabular}{|l|l|l|l|l|}
\hline & \% Abundance Lumen & \% Abundance Duct & Beginning $\mathrm{mM}$ & End $\mathrm{mM}$ \\
\hline $\mathrm{Na}$ & 1.2 & 0.1 & 199 & 16.58 \\
\hline $\mathrm{Cl}$ & 0.1 & 0.5 & 164 & 82 \\
\hline $\mathrm{K}$ & 0.1 & 0.6 & 6 & 36 \\
\hline $\mathrm{PO}_{4}{ }^{--}$ & 0.05 & 0.9 & 55.56 & 1000 \\
\hline $\mathrm{S}$ & 0.1 & 0.35 & 285.71 & 1000 \\
\hline
\end{tabular}

The bioprinting system of Ariadne 1.0 is approximately 50 times the size of the native major ampullate gland of the Nephila clavipes with respect to the diameter of the end of the native gland $(0.004 \mathrm{~mm})$ and the length from the funnel to the end of the spigot (22 mm) (Figure 3) [13]. The system was scaled up to produce a needle diameter of 0.2 $\mathrm{mm}$ because muscle, ligament, and tendon tissue fibers all fall within this diameter range, allowing this prototype to print a wide variety of products $[18,19]$. The Ariadne 1.0 silk-production system maintains an exact ratio to the native gland.

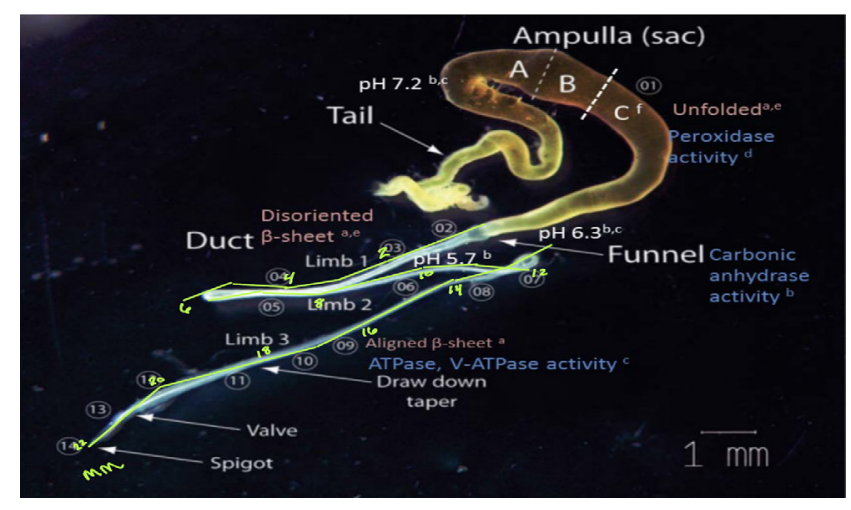

Figure 3. Nephila clavipes ampullate duct demonstrating the length from the funnel to the end of the spigot.

Table 2. Respective dimensions of the Nephila clavipes and Ariadne 1.0 systems based on a scale factor of 50 [13] 


\begin{tabular}{|l|c|l|c|}
\hline \multicolumn{2}{|c|}{ Nephila clavipes } & \multicolumn{2}{c|}{ Ariadne 1.0 } \\
\hline Limb 1 Length $(\mathrm{mm})$ & 6 & IV Tubing Length $(\mathrm{mm})$ & 390 \\
\hline Limb 2 + 3 Length $(\mathrm{mm})$ & 14 & MicroKros Length $(\mathrm{mm})$ & 700 \\
\hline Spigot Length $(\mathrm{mm})$ & 1 & Needle Length $(\mathrm{mm})$ & 50 \\
\hline Total Duct Length $(\mathrm{mm})$ & 22 & Total Chemical Gradient $(\mathrm{mm})$ & 1100 \\
\hline Valve Diameter $(\mathrm{mm})$ & 0.004 & Needle Diameter $(\mathrm{mm})$ & 0.2 \\
\hline
\end{tabular}

This design will provide for exchangeability of the printer needle for future printing projects such as tendon, ligament, and muscle fibers, the printer's needle diameters would be scaled to $0.3,0.4$, and $0.5 \mathrm{~mm}$ respectively), making the scale factors for the printer 75, 100 and 125 times larger than the gland.

The stock dope solution is loaded into a primary syringe and flow through the first IV line of the system. At the first stepped tubing connector, the inner diameter of the system decreases, serving as the start point for the narrowing duct. The second IV has two injection ports that simulate the native chemical conditions of the Nephila clavipes' Limb 1. A potassium phosphate buffer with a $\mathrm{pH}$ of 6.5 is injected to reach a potassium concentration of $36 \mathrm{mM}$ and a phosphate concentration of $1000 \mathrm{mM}$, matching the respective concentrations in the native duct (Table 1) and triggering protein folding. Ammonium sulphate will be added with a sulphur concentration of $1000 \mathrm{mM}$, making the overall printing solution of $\mathrm{pH} 6.3$, the same as the native system (Figure 1 and Table 1). In Limbs 2 and 3 the native major ampullate gland's duct wall behaves like a hollow fiber dialysis membrane, removing water and salts from the liquid silk in order to facilitate proper thread formation and the transition from liquid to solid silk [20]. For Ariadne 1.0 to be proportional to the second and third ducts (totaling $70 \mathrm{~cm}$ ) and match their chemico-physical properties, a $69 \mathrm{~cm}$ hollow filter fiber will be used. The MicroKros ultrafiltration was chosen over other filtration systems due to the selectivity of the hollow filter fibers, which accurately mirror the native silk glands' properties and allow salts, sugars, and water to be filtered out (Figure 4) [21]. Specifically, we will use the SP-10kD MicroKros ultrafiltration system due to its $100 \%$ retention rate of proteins like spider silk that are $70 \mathrm{kD}-700 \mathrm{kD}$ shown in Figure 5 [21]. To speed up the desalting in the hollow filter fiber, we inject isopropanol as a buffer exchange to the liquid silk, and Ajisawa's reagent in a 3:1 volumetric ratio in order to improve the purification process without the need for denaturing processes like heat or multiple passes through the system [22].

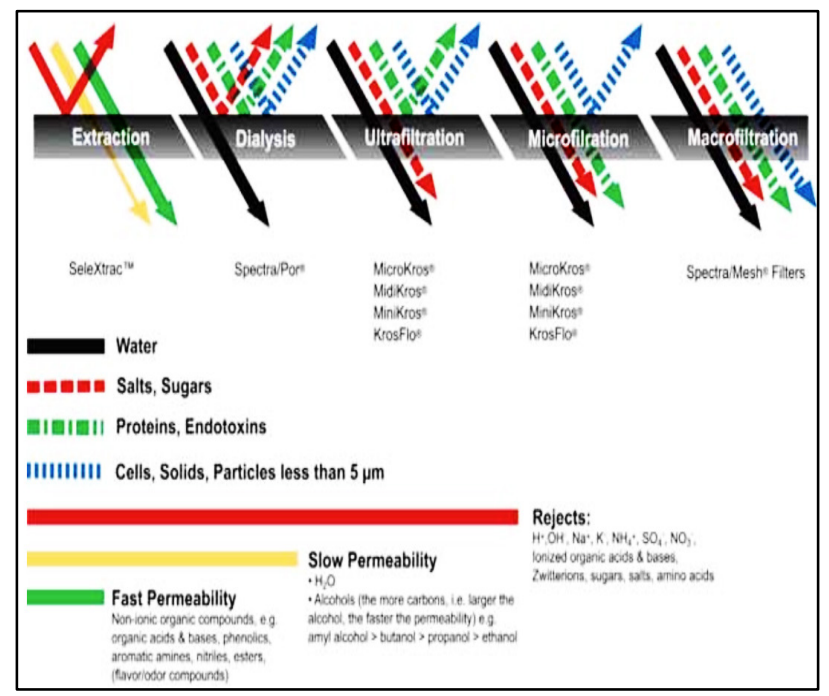

Figure 4. Breakdown of various MicroKros systems featuring different methods of filtration. 


\begin{tabular}{|c|c|c|c|}
\hline \multirow[b]{2}{*}{ Solute Size } & \multicolumn{3}{|c|}{ Retention of Spectrum Membranes (percent) } \\
\hline & SP-10 kD & SP-50 kD & SP-400 kD \\
\hline $\begin{array}{r}1 \mathrm{kd} \\
3 \mathrm{kD} \\
5 \mathrm{kD} \\
10 \mathrm{kD} \\
20 \mathrm{kD} \\
30 \mathrm{kD} \\
40 \mathrm{kD} \\
50 \mathrm{kD} \\
60 \mathrm{kD} \\
100 \mathrm{kD} \\
150 \mathrm{kD} \\
200 \mathrm{kD} \\
300 \mathrm{kD} \\
400 \mathrm{kD}\end{array}$ & $\begin{array}{l}20 \% \\
70 \% \\
90 \% \\
99 \% \\
100 \% \\
100 \% \\
100 \% \\
100 \% \\
100 \% \\
100 \% \\
100 \% \\
100 \% \\
100 \% \\
100 \% \\
\end{array}$ & $\begin{array}{c}0 \% \\
5 \% \\
15 \% \\
30 \% \\
57 \% \\
75 \% \\
85 \% \\
90 \% \\
94 \% \\
99 \% \\
100 \% \\
100 \% \\
100 \% \\
100 \%\end{array}$ & $\begin{array}{l}0 \% \\
0 \% \\
0 \% \\
5 \% \\
16 \% \\
25 \% \\
33 \% \\
41 \% \\
48 \% \\
67 \% \\
80 \% \\
90 \% \\
97 \% \\
99 \%\end{array}$ \\
\hline
\end{tabular}

Figure 5. Desired range of retention rate based on spidroin protein solute size and corresponding MicroKros model.

\section{Printing Mechanisms}

To ensure Ariadne 1.0's entire system is easily modifiable and inexpensive, a Monoprice V2 mini filament extrusion printer was altered to print with biological materials. The conversion of a printer in this manner required an extensive redesign of many components - specifically the printer head and filament extruder assemblies. Bio gel use requires maintenance of constant pressure throughout the feeding and printing process. This was accomplished by replacing all original mechanical extrusion components with a multi-stage, trans-energy-domain, mechatronic system. The filament rack and filament feeder were removed and replaced with a large syringe to house the spider-dope solution. The motor from the original extruder was repurposed to drive the syringe assembly. Utilizing electrical input from the printer, the motor's controlled rotational motion drives the translation of the plunger, providing hydraulic pressure to the solution in the syringe. This method allows the user to vary the diameter of the silk-fiber product by directly controlling the volumetric flow rate of the solution through the system. The printer head was designed to securely mount to the $\mathrm{x}$-axis of the printer and allow for high precision, low vibrational extrusion with a fine-point needle. The hydraulic extrusion line ends in a $10 \mathrm{~mL}$ syringe with a small diameter glass capillary tube for a needle tip. The tubing connection from the chemical gradient to the final syringe extruder was designed to be both secure and interchangeable. The entire syringe component can be removed from the printer head and replaced with an alternate, allowing for customization of the needle tip and size of the extruder. We control the printer's electronic components using an Arduino microcontroller system, as they are widely accessible and intuitive to program and design. The Ariadne 1.0 will give the user complete control over the feed rate, printing speed, travel rate, needle diameter, and temperature of the printing process to support many different silk applications.

\section{Documentation of Final Prototype}

The Ariadne 1.0 prototype expands on a low-cost bioprinter developed by UC Davis students in the Bio Innovation Group. Their Bioprinter team modified a plastic filament printer to print organic hydrogel with a hydraulic extrusion assembly. The base model for the build is a Monoprice Mini V2, which is affordable and widely available. 3D-OPS replicated the low-cost bioprinter (Figure 6a), then modified it further to simulate the chemically necessary processes to extrude spider silk (Figure 6b). The schematics of the UC Davis Bioprinter team provided a baseline design, but 3D-OPS designed or modified the features discussed in this section. 


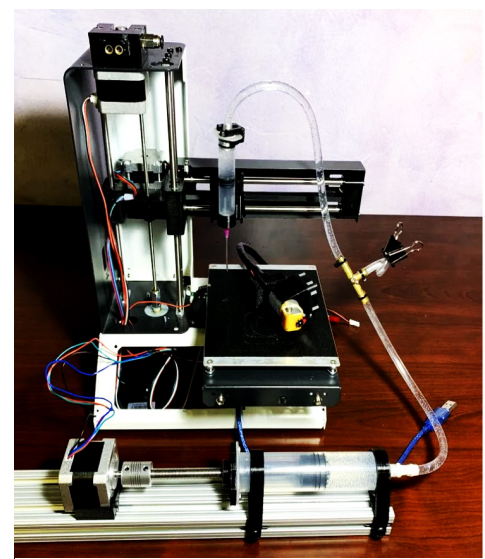

$\underline{1 a}$

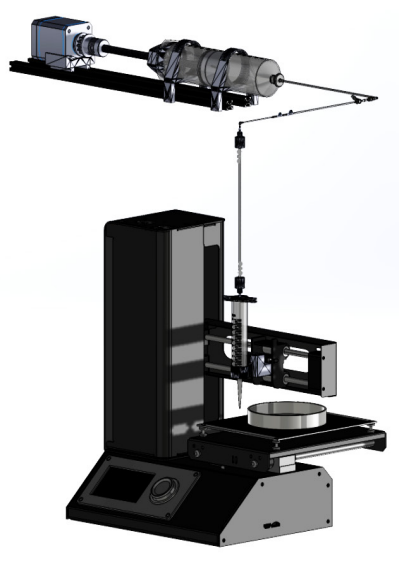

$6 \mathrm{~b}$

Figure 6. Bioprinter prototypes. Figure 6a depicts the UC Davis Bioprinter Team design and initial build from https://www.instructables.com/Low-Cost-Bioprinter/. Figure 6b depicts the 3D-OPS Ariadne 1.0 prototype rendered in SOLIDWORKS.

Due to the COVID-19 pandemic, 3D-OPS could not resume normal lab activities to complete the physical prototype of Ariadne 1.0. However, all components have been CAD modeled and assembled into dimensionally and mechanically correct parts. Figure 7 is a color-coded diagram of Ariadne 1.0, accompanied by the corresponding part key.

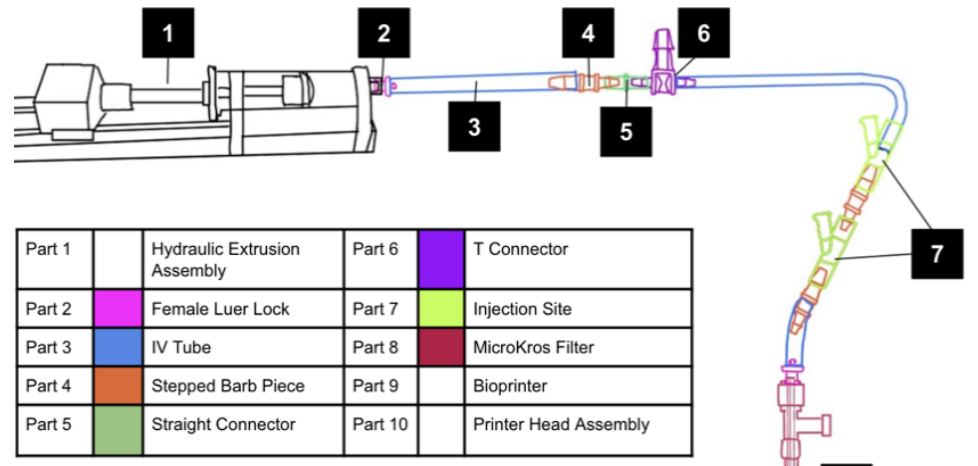

8

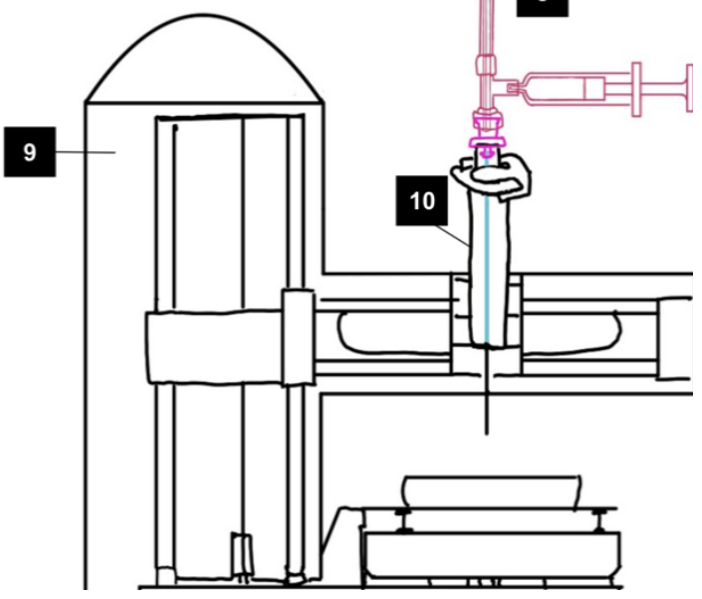


Figure 7. Color coded diagram and legend of Ariadne 1.0 assembly parts. Note: Only one of the four Extruder assemblies is represented in the diagram.

Part 1 corresponds to the hydraulic extrusion sub-assembly and is the first step in the printing process. A $100 \mathrm{~mL}$ syringe filled with $60 \mathrm{~mL}$ of spider dope solution is secured to a $200 \mathrm{~mm}$ Maker Beam XL bar track. The syringe is capped with a specially designed lid that securely screws to the brim of the syringe, into which an $8 \mathrm{~mm} x 2 \mathrm{~mm}$ threaded rod is inserted. One end of the rod is epoxied to a modified syringe plunger. The other is coupled to a freely sliding step motor in Extruder 1. The motor was repurposed from the Monoprice filament extruder and receives power and data from the printer. The controlled rotation of the motor drives the translation of the plunger, providing hydraulic pressure to the solution in the syringe. The desired fiber extrusion rate ranges from $0.5-30 \mathrm{~m} / \mathrm{min}$ depending on the application, requiring a volumetric feed rate of $15.7-935 \mu \mathrm{L} / \mathrm{min}$ from the $100-\mathrm{mL}$ syringe. The necessary angular velocity of the motor can be calculated from the desired flow velocity within these ranges. A similar system of coupled motor and syringe adds chemical solutions to the IV line at a controlled rate.

Parts 2-5 facilitate secure connections between different phases of the Chemical Gradient. Part 6 is a Tconnector used at the high point of the line to evacuate air bubbles. At the first injection site (Part 7a), the step motor in the Extruder 2 assembly pumps potassium phosphate solution from a 100mL syringe into the chemical gradient line to increase the potassium and phosphorus concentration to $36 \mathrm{mM}$ and $1000 \mathrm{mM}$, system respectively. At the second injection site (Part 7b), Extruder 3 adds ammonium sulfate to increase sulfur to $1000 \mathrm{mM}$.

Figure 8 shows where the acidic solution enters the MicroKros SP-010 Filter (Part 8) through Port 1. This device consists of parallel hollow-fiber membranes in a polysulfone housing that utilizes tangential flow to filter microparticles while maintaining high permeation, low waste retention, and laminar flow behavior. At Port 2, Extruder 4 pumps isopropanol from a 200-mL syringe into the solution such that a constant 3:1 ratio of isopropanol to silk fibroin solution is maintained. Water and salts will pass through the tangential filter membrane and into the $100-\mathrm{mL}$ collection syringe of Port 3, leaving a purified and dehydrated silk retentate that exits through Port 4.

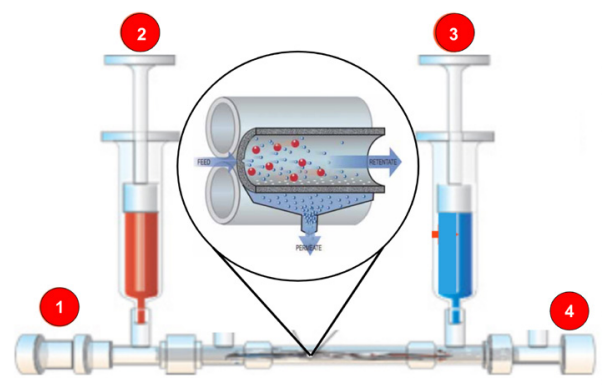

Figure 8. MicroKros SP-010 Filter with labeled ports and blown-up cross-sectional diagram

Part 9 correlates to the Monoprice printer body that houses an Arduino Mega 2560 board, Z-axis motors, and wiring. The frame and axis motion are unaltered from the stock printer, but all electronics within the printer are controlled using the Arduino microcontroller and Arduino IDE firmware. This allows the user to control the feed rate of the spider filament using Extruder 1 and to alter the travel speed of the $\mathrm{X} / \mathrm{Y} / \mathrm{Z}$ axes and the heated bed temperature without modifying the printer's G-code.

The printer-head sub-assembly (Part 10) is mounted to the X-axis carriage. A slotted extension fastened to the carriage creates a dovetail joint for easy syringe assembly removal (Figure 9). A custom syringe mount can be slid into place and optionally secured with a U-shaped bracket. The mount holds a $10-\mathrm{mL}$ syringe that functions as the printer head for this system. The end of the chemical gradient line feeds directly into a capillary glass tube suspended in place within the syringe using a flexible silicone mold. The mold provides insulation and stabilization from travel motion of the printer head. For applications requiring silk filament of different diameters, the entire syringe head can 
be slid out of the mount and replaced with an alternate syringe featuring a different glass capillary tube. The current capillary tube has an inner diameter of $0.02 \mathrm{~mm}$, which is the optimal silk fiber thickness for printing fibrous tissue. A specialized cap on the syringe connects the tubing to the glass capillary and holds the tube securely in place while the printer head travels. The cap may be removed to interchange the size of the tubing and is clamped to the brim of the syringe with screws. The printer head assembly allows for 3-axis travel and extrusion while maintaining constant pressure throughout the chemical gradient line and minimizing the impact of motion on the proteins. Because the syringe assembly is longer than the original extruder head of the Monoprice, a spacer was mounted to the $\mathrm{z}$-axis motor to prematurely trigger the $\mathrm{z}$-axis switch when the syringe tip nears the printer bed.

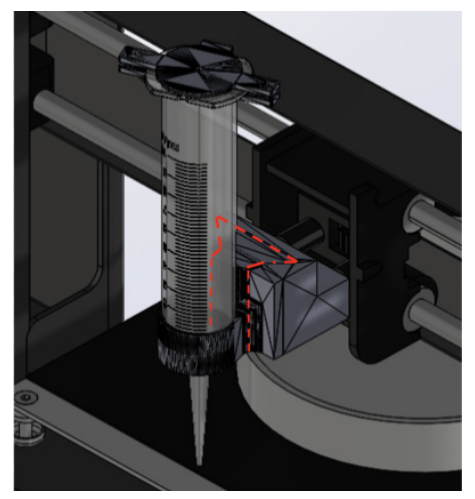

Figure 9. Specialized syringe hydraulic extrusion printer head mounted to the $\mathrm{x}$-axis carriage using a dovetail joint.

A comprehensive wiring diagram including additional external stepper motors is depicted in Figure 10. The $\mathrm{X} / \mathrm{Y} / \mathrm{Z}$-axes motors from the Monoprice are rewired to communicate with the Arduino board, as are other electronic components, including the heated build plate and thermistor. Extruder 1 corresponds to the motor driving the plunger of the 100-mL syringe in the hydraulic extrusion sub-assembly. The port to the right of Extruder 1 hosts an extension which connects to Extruder 2 (which drives the injection of potassium phosphate into the chemical gradient), Extruder 3 (which drives the injection of ammonium sulfate into the chemical gradient), and Extruder 4 (which drives the injection of isopropanol into the chemical gradient).

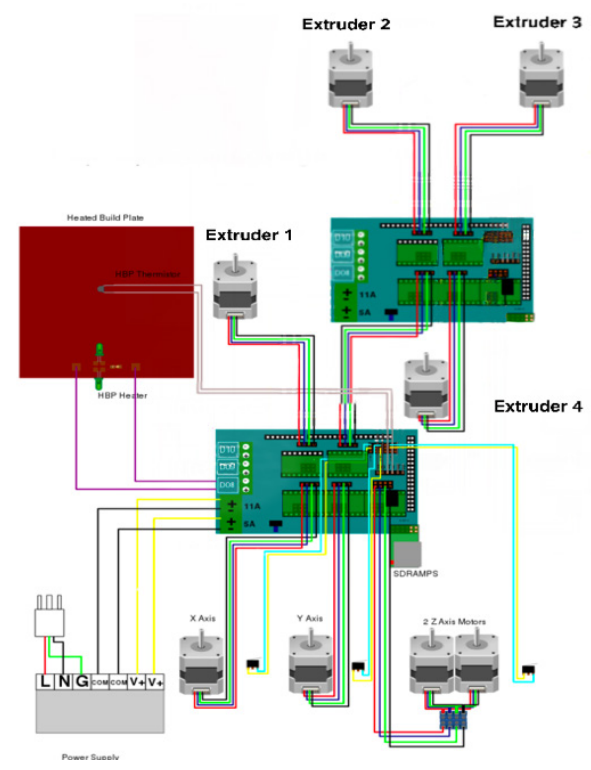


Figure 10. Comprehensive wiring diagram depicting Arduino Mega boards, stepper motors, and printer components.

\section{Results of a Search for Prior Art, Assessment, and Patentability}

The relevant patents in Table 3 include claim elements regarding the aggregation of silk proteins and the production of silk fibers. Ariadne 1.0 aims to produce silk fibers, but its novel design separates it from the prior art. In contrast to relevant patents, Ariadne 1.0 operates on regulating heavily conserved N-Terminal and C-Terminal domains instead of spidroin repetitive motifs, enabling selection from numerous fibroin proteins [7]. Ariadne 1.0's dynamic system also allows the individual alteration of various inputs such as concentrations of ions, $\mathrm{pH}$ gradient values, protein weight-per-volume ratios, and flow rate, reshaping the product to fit the individualized needs. Someone skilled in the art would view our device as non-obvious and therefore patentable.

Table 3: Relevant patents including claim elements regarding the aggregation of silk proteins and the production of silk fibers.

\begin{tabular}{|l|l|l|}
\hline & Patent Number & Analysis \\
\hline 1 & US20190062557A1 & $\begin{array}{l}\text { Claims describe a method of concentrating a solution of silk protein and protein } \\
\text { denaturant to a concentration of at least 10\% w/v, and then producing silk-spinning } \\
\text { dope by drawing/extrusion. }\end{array}$ \\
\hline 2 & US007951908B2 & $\begin{array}{l}\text { Claims present a method of aggregating spider silk proteins through acidification, } \\
\text { potassium phosphate application, or mechanical stress. }\end{array}$ \\
\hline 3 & US008721991B2 & $\begin{array}{l}\text { Claims surround a 3-channel microfluidic device that utilizes a laminar flow to } \\
\text { aggregate spider silk proteins and assemble silk. }\end{array}$ \\
\hline 4 & US20110201783A1 & $\begin{array}{l}\text { Claims describe an apparatus that employs phosphate and potassium ions to separate } \\
\text { out protein-rich regions of a silk solution, which are then drawn out to produce a } \\
\text { thread. }\end{array}$ \\
\hline 6 & US20130225476A1 & $\begin{array}{l}\text { Claims are more focused on the nucleic acid and amino acid sequences involved in the } \\
\text { production of a biopolymer with high tensile strength, extensibility, and toughness. }\end{array}$ \\
\hline 7 & US20140035975A1 & $\begin{array}{l}\text { Claims present a device that allows for the controlled printing of structures using a jet } \\
\text { made up of an outer sheath liquid and inner viscoelastic liquid. }\end{array}$ \\
\hline 8 & US20040102614A1 & $\begin{array}{l}\text { Claims introduce methods and apparatuses that utilize 5-50\% spider silk protein dope } \\
\text { for spinning silk fibers. }\end{array}$ \\
\hline
\end{tabular}

Search Terms Used: (3d printer) AND (spider silk); (artificial spider silk) AND (artificial tissue); (spinning) AND (printing); (extrusion printer); (chemical gradient) AND (mechanical); (natural silk)

Ariadne 1.0 has little marketplace competition as no commercial devices currently create artificial tissue from spider silk. The most similar designs are artificial bone grafts and, to a lesser degree, surgical meshes. The former is far more expensive; both pose several risks and challenges and are ultimately inadequate in treating most musculoskeletal conditions.

\section{Anticipated Regulatory Pathway}


Given the lack of significant risks and the similarity in intended application to current class II approved devices involved in tissue engineering or reinforcement, such as synthetic/polymeric surgical meshes and artificial scaffolding, Ariadne 1.0's system falls under the FDA class II biomedical device [23]. However, the novelty of Ariadne 1.0's design raises questions about its effectiveness and safety, compromising any attempt at showing substantial equivalency to a predicate device [24]. Consequently, Ariadne 1.0 is not eligible for 510(k) approval and will require premarket approval (PMA).

The FDA has already approved numerous comparable skin and musculoskeletal treatments through PMA [25]. However, ReGen Biologics' Collagen Scaffold, another similar device, initially failed to demonstrate the safety and effectiveness and consequently was denied PMA by the FDA [26]. After several appeals, allegations of unfair treatment by the panel, and updated data, the FDA reversed its decision and cleared ReGen's device via 510(k) [27]. Ultimately, Ariadne 1.0's system and product would need to undergo rigorous experimentation and clinical trials to demonstrate safety and effectiveness to gain PMA successfully. Ariadne 1.0 will be overseen by the Center for Biologics Evaluation and Research (CBER) because it is a biological therapeutic product [28].

\section{Reimbursement}

Medicare and Medicaid will likely partially or entirely cover the Ariadne 1.0 system if the surgeries are considered medically necessary; coverage depends on the surgery type and provider. Medicare and Medicaid will guarantee full coverage of the Ariadne 1.0 product in lower extremity joint replacement surgeries (LEJR) through their Comprehensive Care for Joint Replacement (CJR) bundle. The CJR bundle provides beneficiaries with a comprehensive plan covering all inpatient or outpatient admission services through 90 days post-discharge [29,30]. For all other medically necessary surgeries, Medicare B offers $80 \%$ coverage of total fees, which would include the Ariadne 1.0 system.

Total medical fees and coverage for Medicaid enrollees depend on location as some states' waivers allow for higher premiums and cost-sharing than federal rules permit [31]. However, nationwide, Medicaid prevents out-ofpocket costs exceeding $5 \%$ of family income. Due to the variability of fees and coverage state-to-state, Ariadne 1.0's Medicaid coverage for non-LEJR surgeries is unknown.

\section{Estimated Manufacturing Costs}

Table 4. Detailed list of all manufacturing costs associated with the prototype. Mechanical part costs are per unit. Chemical costs represent the volumes for creating stock solutions; usage varies depending on the application. 


\begin{tabular}{|c|c|c|c|c|c|c|c|}
\hline Material & $\begin{array}{l}\text { Price per } \\
\text { unit }\end{array}$ & Quantity & $\begin{array}{l}\text { Total } \\
\text { Price }\end{array}$ & Material & $\begin{array}{l}\text { Price per } \\
\text { unit }\end{array}$ & Quantity & $\begin{array}{l}\text { Total } \\
\text { Price }\end{array}$ \\
\hline $\begin{array}{l}\text { Monoprice V2 3D } \\
\text { Printer }\end{array}$ & $\$ 200$ & 1 & $\$ 200$ & $.2 \mathrm{~mm}$ ID Glass Capillary & $\$ 0.53$ & 1 & $\$ 0.53$ \\
\hline $\begin{array}{l}\text { 3D Printer Controller } \\
\text { Kit }\end{array}$ & $\$ 39.99$ & 1 & $\$ 39.99$ & Straight Connector Piece & $\$ 0.07$ & 2 & $\$ 0.15$ \\
\hline Arduino ProtoShield & $\$ 9.99$ & 1 & $\$ 9.99$ & T-Connector & $\$ 0.77$ & 1 & $\$ 0.77$ \\
\hline PLA Filament & $\$ 22.99$ & 1 & $\$ 22.99$ & Injection Site/ Y-tube & $\$ 0.85$ & 2 & $\$ 1.70$ \\
\hline Maker Beam & $\$ 4.12$ & 8 & $\$ 33.00$ & $\begin{array}{l}\text { Microkros Hollow Fiber } \\
\text { Filter }\end{array}$ & $\$ 167$ & 1 & $\$ 167.00$ \\
\hline $\begin{array}{l}\text { M3 screws assorted } \\
\text { sizes }\end{array}$ & $\$ 12.99$ & 1 & $\$ 12.99$ & Luer Cap (Male) & $\$ 0.14$ & 1 & $\$ 0.14$ \\
\hline 8-mm threaded rod & $\$ 7.99$ & 4 & $\$ 31.96$ & $\begin{array}{l}\text { Silicone Mold Making } \\
\text { Kit }\end{array}$ & $\$ 36.97$ & 1 & $\$ 36.97$ \\
\hline 608RS Ball Bearing & $\$ 0.92$ & 4 & $\$ 3.67$ & $500 \mathrm{~g} \mathrm{CaCl}_{2}$ & $\$ 8.25$ & 1 & $\$ 8.25$ \\
\hline $\begin{array}{l}100-\mathrm{mL} \text { Syringe and } \\
\text { tubing }\end{array}$ & $\$ 3.99$ & 4 & $\$ 15.98$ & $\begin{array}{l}500 \mathrm{~mL} \text { Ethanol Lab } \\
\text { Grade }\end{array}$ & $\$ 19.99$ & 1 & $\$ 19.99$ \\
\hline 10-mL Syringe & $\$ 0.17$ & 1 & $\$ 0.17$ & 1 Gal Pure Water & $\$ 15.99$ & 1 & $\$ 15.99$ \\
\hline 200-mL Syringe & $\$ 7.99$ & 1 & $\$ 7.99$ & $2.5 \mathrm{~kg} \mathrm{NaHCO}_{3}$ & $\$ 14.95$ & 1 & $\$ 14.95$ \\
\hline Zip Ties (100 pieces) & $\$ 11.99$ & 1 & $\$ 11.99$ & $500 \mathrm{~g} \mathrm{Na}_{2} \mathrm{CO}_{3}$ & $\$ 19.99$ & 1 & $\$ 19.99$ \\
\hline $\begin{array}{l}\text { Jumper Cables (120 } \\
\text { pieces) }\end{array}$ & $\$ 5.78$ & 1 & $\$ 5.78$ & $500 \mathrm{~g} \mathrm{NaCl}$ & $\$ 9.95$ & 1 & $\$ 9.95$ \\
\hline $\begin{array}{l}\text { M3 Heat Seat Nuts } \\
2 \mathrm{~mm} \text { width }\end{array}$ & $\$ 0.47$ & 4 & $\$ 3.76$ & $500 \mathrm{~g}$ Urea & $\$ 10.15$ & 1 & $\$ 10.15$ \\
\hline Wire Wrap & $\$ 13.99$ & 1 & $\$ 13.99$ & $\begin{array}{l}1 \mathrm{lb} \text {. Potassium } \\
\text { Dihydrogen Phosphate }\end{array}$ & $\$ 21.99$ & 1 & $\$ 21.99$ \\
\hline $\begin{array}{l}\text { MakerBeam XL } \\
\text { M3x6 Bolts }\end{array}$ & $\$ 0.07$ & 16 & $\$ 1.12$ & $\begin{array}{l}500 \mathrm{~g} \text { Potassium } \\
\text { Phosphate }\end{array}$ & $\$ 28.90$ & 1 & $\$ 28.90$ \\
\hline $\begin{array}{l}\text { MakerBeam XL } \\
\text { M3x6 Nuts }\end{array}$ & $\$ 0.32$ & 16 & $\$ 5.12$ & $1 \mathrm{lb}$. Ammonium Sulfate & $\$ 8.99$ & 1 & $\$ 8.99$ \\
\hline Female Luer Lock & $\$ 0.46$ & 3 & $\$ 1.38$ & 1 Gal Isopropanol & $\$ 39.00$ & 1 & $\$ 39.00$ \\
\hline \multirow[t]{2}{*}{ Stepped Barbed Tube } & $\$ 0.82$ & 3 & $\$ 2.46$ & & & & \\
\hline & & & & & & Total & $\$ 830$ \\
\hline
\end{tabular}

When manufacture of the Ariadne 1.0 system is scaled up, volume discounts may apply to the chemical gradient components and chemicals used. The cost of silk fibroin proteins varies considerably based on the desired source type and is not to be factored into the manufacturing cost of the system. The required software is free to the user and includes CAD software, Arduino IDE, Repetier Host, and Ultimaker Cura 4. Ariadne 1.0 will be developed in a lab environment and marketed to individuals and organizations already established within the medical field, such as hospitals and research centers. Because of this, we assume the user is working in an environment where they have access to standard lab equipment such as PPE and measurement devices.

Quality assurance testing can be incorporated into each stage of the process. During the pre-printing process, the spider dope can be tested to ensure the desired viscosity, protein orientation, and solution purity. During printing, the $\mathrm{pH}$ of the chemical gradient can be measured using a $\mathrm{pH}$ meter, the temperature can be verified using thermocouples, and the flow rate can be monitored with a mass flow meter. The product can be analyzed by printing a small 
sample and testing it for tensile strength, elasticity, and purity of the proteins. As previously disclosed, 3D-OPS has been unable to test the prototype in laboratory conditions; therefore, quality assurance metrics have not been instituted and can not be factored into manufacturing cost at this time.

\section{Potential Market and Impact}

Some of the potential medical applications of spider silk include stronger micro-sutures, sturdy bio-scaffolds for artificial skin and nerve grafts, tendon and ligament repair with improved strength and elasticity, weight-bearing artificial knee menisci, and liquid silk to treat wounds [32]. The antimicrobial nature of spider silk is especially promising in its potential to combat gram-negative bacteria, which poses a significant threat to human health due to its antibiotic resistance. Spider silk is conductive, making it a good candidate for nerve repair, and complementing the good tensile strength.

Patients with musculoskeletal injuries that need muscle, tendon, and ligament repair/replacement and providing surgeons will be the primary users of Ariadne 1.0's silk product. In 2004, musculoskeletal injuries affected an estimated 33 million people in the United States, with tendon, ligament, and joint capsular injuries accounting for over half of those [29]. Achilles tendinosis repair, anterior cruciate ligament restoration, and other soft-tissue repair operations are also common. According to statistics from 2020, the global market for orthopedic soft tissue repair is expected to increase at a compound annual growth rate of 6.2 percent to $\$ 10.3$ billion by 2027 [32].

Current commercially available 3D bioprinters generally cost between $\$ 10,000-150,000$ [33]. This cost gets passed down to the users, preventing low-income hospitals and uninsured patients from accessing the latest technology in the medical field. In addition to their high cost, 3D bioprinters in the current market have limited capacity for customization, complicated maintenance, and operating systems that are difficult to use without an expert staff [33]. By contrast, the Ariadne 1.0 bioprinter can be affordably manufactured using accessible parts, provides for customization for various applications, and is intuitive to use.

We estimate that the entire artificial silk system, including our software, hardware, and biological additions, will cost approximately $\$ 830$ per unit based on the current cost of materials. Medical equipment stores, hospitals, and research laboratories would be our primary clients and distribution routes. To maximize economic feasibility and medical effectiveness, we aim to keep pricing below $\$ 1,000$. Ariadne 1.0's silk solution does not use any caustic chemicals, minimizing risk in the pre-printing solution preparation. The pre-printing process can also be scaled up industrially to create bulk amounts of stable protein solution. Silk can withstand a wide variety of environmental conditions without degrading, lowering accessibility barriers based on the lack of highly regulated lab conditions. This pre-printing solution can also be used with an array of fibroin proteins, paving the way for a better understanding of their properties, and implementing them in new medical applications. Consequently, this lower price combined with a fabrication method that does not require a sophisticated lab environment and doesn't produce a caustic by product will provide more patients greater accessibility to this technology, result in faster recovery, and enable safer, shorter procedures of artificial silk-based tissue grafts compared to traditional allograft therapies.

The growing prevalence of 3D printers as medical devices broadens the field of possibilities for artificial tissue generation. Existing 3D imaging systems can already generate highly accurate models of a patient's body. 3D imaging and 3D printing compatibility enable efficient patient care and faster turnaround times for tissue construction. Moving toward a more computerized field of medicine also enables stress and fatigue analysis to be run on any printed structures to ensure the safety and reliability of the implant. Pairing this technology with a device like the Ariadne 1.0 could allow providers to produce customized musculoskeletal replacements or repair parts based on a patient's physicality [34]. 


\section{References}

[1] United States Bone and Joint Initiative: The Burden of Musculoskeletal Diseases in the United States (BMUS), Third Edition. 2014. http://www.boneandjointburden.org.

[2] Römer L, Scheibel T. The elaborate structure of spider silk. Prion. 2008; 2(4), 154-161. https://doi.org/10.4161/pri.2.4.7490

[3] Yang G, Rothrauff B, Tuan R. Tendon and ligament regeneration and repair: Clinical relevance and developmental paradigm. Birth Defects Research Part C: Embryo Today: Reviews. 2013. 99(3), 203-222. https://doi.org/10.1002/bdrc.21041

[4] Salehi S, Koeck K, Scheibel T. Spider Silk for Tissue Engineering Applications. Molecules. 2020; $25(3), 737$. https://www.ncbi.nlm.nih.gov/pmc/articles/PMC7037138/

[5] Chhabra K, Sheetz K, Nuliyalu U, Dekhne M, Ryan A, Dimick J. Out-of-Network Bills for Privately Insured Patients Undergoing Elective Surgery With In-Network Primary Surgeons and Facilities. JAMA. 2020; 323(6), 538. https://doi.org/10.1001/jama.2019.21463

[6] Benefits and associated risks of using allograft, autograft and synthetic bone fusion material for patients and service providers - A Systematic Review. JBI Library of Systematic Reviews. 2020; 8(Supplement), 1-13. https://doi.org/10.11124/jbisrir-2010-851

[7] Rising A, Johansson J. Toward spinning artificial spider silk. Nat Chem Biol. 2015; 11, 309-315. https://doi.org/10.1038/nchembio.1789

[8] Pusch K, Hinton T, Feinberg A. Large volume syringe pump extruder for desktop 3D printers. HardwareX. 2018; Volume 3, Pages 49-61, ISSN 2468-0672, https://doi.org/10.1016/j.ohx.2018.02.001.

[9] Mohtar J, Ooi W, Yusuf F. Spider Silk Processing for Spidroin Recovery from Crossopriza Lyoni Web. IOP Conference Series: Materials Science and Engineering. 2018; vol. 318, p. 012016. doi: $10.1088 / 1757$ 899x/318/1/012016.

[10] M Andersson. Carbonic Anhydrase Generates CO2 and H+ That Drive Spider Silk Formation Via Opposite Effects on the Terminal Domains. PLOS Biology. 2014; vol. 12, no. 8, pp. 1-14. doi:10.1371/journal.pbio.1001921.

[11] Clark J. pH curves (titration curves). ChemGuide. 2013. https://www.chemguide.co.uk/physical/acidbaseeqia/phcurves.html

[12] Gaines W, Sehorn M, Marcotte W. Spidroin N-terminal Domain Promotes a pH-dependent Association of Silk Proteins during Self-assembly. Journal of Biological Chemistry. 2010; vol. 285, no. 52, pp. 40745-40753. doi: 10.1074/jbc.M110.163121.

[13] Peng, C. Creating Biomaterials from Plant-derived Recombinant Spider Silk-like Proteins. Tiger Prints. 2016. https://tigerprints.clemson.edu/all dissertations/1824 
[14] Mu X, Fitzpatrick V, Kaplan D. From Silk Spinning to 3D Printing: Polymer Manufacturing using Directed Hierarchical Molecular Assembly. Advanced Healthcare Materials. 2020; vol. 9, no. 15, p. 1901552. doi: https://doi.org/10.1002/adhm.201901552.

[15] Finnigan W. The effect of terminal globular domains on the response of recombinant mini-spidroins to fiber spinning triggers. Scientific Reports. 2020; vol. 10, no. 1, p. 10671. doi:10.1038/s41598-020-67703-1.

[16] Mohammadi $P$. Controllable coacervation of recombinantly produced spider silk protein using kosmotropic salts. Journal of Colloid and Interface Science. 2020; vol. 560, pp. 149-160. doi: https://doi.org/10.1016/j.jcis.2019.10.058.

[17] Zheng Z. Lithium-free processing of silk fibroin. J Biomater Appl. 2016; vol. 31, no. 3, pp. 450-463. doi: $10.1177 / 0885328216653259$.

[18] Erisken C, Zhang X, Moffat K, Levine W, Lu H. Scaffold fiber diameter regulates human tendon fibroblast growth and differentiation. Tissue Eng Part A. 2013; vol. 19, no. 3-4, pp. 519-528. doi:10.1089/ten.tea.2012.0072.

[19] Noonan A, Zwambag D, Mazara N, Weersink E, Power G, Brown S. Fiber Type and Size as Sources of Variation in Human Single Muscle Fiber Passive Elasticity. Journal of Biomechanical Engineering. 2020; vol. 142, no. 8. doi: $10.1115 / 1.4047423$.

[20] Wu H, Wu S, Yang T, Yang J. A Facile Measurement for Monitoring Dragline Silk Dope Concentration in Nephila pilipes upon Spinning. Materials. 2018; vol. 11, no. 10. doi:10.3390/ma11101951.

[21] ABC's of Filtration \& Bioprocessing. yumpu.com. Spectrum Laboratories, Inc. https://www.yumpu.com/en/document/read/28617071/abcs-of-filtration-bioprocessing-spectrum-laboratories-inc

[22] Zhang M, Weng Y, Zhang Y. Accelerated desalting and purification of silk fibroin in a CaCl2-EtOH-H2O ternary system by excess isopropanol extraction. Journal of Chemical Technology \& Biotechnology. 2021; vol. 96, no. 5, pp. 1176-1186. doi: https://doi.org/10.1002/jctb.6629.

[23] CFR - Code of Federal Regulations Title 21. Department of Health and Human Services. Food and Drug Administration. 2020. https://www.accessdata.fda.gov/scripts/cdrh/cfdocs/cfcfr/cfrsearch.cfm?fr=878.3300

[24] Health R. Premarket Notification 510(k). FDA. 2020. https://www.fda.gov/medical-devices/premarket-submissions/premarket-notification-510k

[25] Hellman K. Tissue Engineering: Translating Science to Product. Topics in Tissue Engineering. 2008; vol. 4. https://www.oulu.fi/spareparts/ebook topics in $\mathrm{t}$ e vol4/abstracts/hellman.pdf

[26] "Testimony on Collagen Scaffold Medical Device," Public Citizen. Nov. 14, 2008. https://www.citizen.org/article/testimony-on-collagen-scaffold-medical-device/

[27] Review of the ReGen Menaflex ${ }^{\circledR *}$ : Departures from Processes, Procedures, and Practices Leave the Basis for a Review Decision in Question. FDA; 2009. p. 33. https://www.fda.gov/media/77734/download 
[28] Leon D. FDA Premarket Regulation of Tissue-Engineered Replacement Parts for Humans. Digital Access to Scholarship at Harvard Library. 2003. Available: https://dash.harvard.edu/handle/1/8965598

[29] Comprehensive Care for Joint Replacement Model. Cms.gov. Center for Medicare and Medicaid Services. 2016. https://innovation.cms.gov/innovation-models/cjr

[30] Hartmann D. CMS finalizes rule to expand and modify Comprehensive Care for Joint Replacement Model. Health Industry Washington Watch. 2021. https://www.healthindustrywashingtonwatch.com/2021/05/articles/department-of-health-and-human-services/cms-finalizes-rule-to-expand-and-modify-comprehensive-care-for-jointreplacement-model/

[31] Rudowitz R, Garfield R, Hinton E. 10 Things to Know about Medicaid: Setting the Facts Straight. Kaiser Family Foundation. 2020. https:/www.kff.org/medicaid/issue-brief/10-things-to-know-about-medicaid-setting-the-factsstraight/?gclid=CjwKCAjwzMeFBhBwEiwAzwS8zJ7rbaFVMeQ5K2jnseMm5qZZ0pERpyxwlWAXZR OTspN14ti8kJdbhoCse8QAvD BwE

[32] Jain A. Spider Silk in Medicine. Young Scientists Journal. 2015. https://archive.ysjournal.com/article/spidersilk-in-medicine/

[33] Ioannidis K. A Custom Ultra-Low-Cost 3D Bioprinter Supports Cell Growth and Differentiation. Frontiers. 2020. https://doi.org/10.3389/fbioe.2020.580889

[34] Shriners Hospitals for Children. Innovative imaging technology provides 3D assessment for scoliosis. News and Events - Shriners Hospitals for Children. 2021. https://www.shrinershospitalsforchildren.org/shc/newsevents/innovative-imaging-technology-provides-3d-assessme-1348 